\title{
Optimal Transform in Perceptually Uniform Color Space and Its Application in Image Retrieval*
}

\author{
Ying Chen Pengwei Hao \\ Center for Information Science, Peking University, Beijing, 100871, China \\ Department of Computer Science, Queen Mary, University of London, E1 4NS, UK \\ E-mail: \{chenying, phao\}@cis.pku.edu.cn
}

\begin{abstract}
To find an appropriate color transform is necessary and helpful for the applications of color images to extract color features or to reduce the data redundancy. In this paper, we proposed a new scheme to find color transforms close to the optimal transform and agree with human vision system for comparison. We first apply the perceptually uniform color space transform to all the hundreds color test images to convert RGB values into uniform CIE LAB components, and then use principal component analysis (PCA) in the uniform space to find the image-dependent optimal color transforms (KLT). In our experiments, we have obtained a new color space: an approximate but fixed transform in CIE LAB space, namely LAR space, which is just the LAB rotated and has an elegant and simple form. The proposed LAR transform performs better than RGB, YCbCr, YUV, HSV and LAB in color-based image retrieval.
\end{abstract}

\section{Introduction}

Color images are everywhere in science, technology, medicine and industry. Color images are acquired and reproduced based on tristimulus values whose spectral composition is carefully chosen according to the principles of color science. Color space transform is critical for color feature extraction and data redundancy reduction. To find an appropriate color transform is necessary and very helpful in many color image applications, such as image display, processing, retrieval, recognition, and compression.

In 1931, Commission Internationale de l'Eclairage (CIE) defined three standard primaries, called $\mathrm{X}, \mathrm{Y}$ and $\mathrm{Z}$, to replace red, green and blue (RGB) and with positive weights to match all the colors we see. It took CIE a decade or more to find a transformation of CIE XYZ into a reasonable perceptually uniform space. So far, CIE standardized two perceptually uniform systems, CIE LUV and CIE LAB. Besides, many color standards enable users to have the freedom to choose the color space in which to represent their data. RGB, CMYK, YIQ, HSV, CIE 1931 XYZ, CIE LUV, CIE LAB, YES, CCIR 601-2 YCbCr, and SMPTE-C RGB are proposed for diverse requirements [1]. However, in many applications, we need

* Supported by FANEDD China, under Grant 200038. some appropriate color transforms and we also wish the transformed or the inverse-transformed components are inter-comparable and the comparison done by computers is the same as we do by our human visual system. Therefore, we need compare the results in a perceptually uniform color space after applying inverse of our specific color space transforms. Our idea is to find some optimal color transforms in the uniform space.

In this paper, a new scheme to find an optimal color transform is proposed. We transform color images into three components in the uniform space CIE LAB, and use principal components analysis to find image-dependent optimal color transform, Karhunen-Loeve Transform (K-L transform, or KLT). Then, we take the transform as an image-independent color transform and apply it to image retrieval.

\section{Principal Components Analysis in CIE LAB Color Space}

The conversion from RGB color values into CIE LAB components is implemented by converting from nonlinear RGB space into linear RGB space first, then into CIE XYZ space, and finally into CIE LAB space [2].

A system using linear interpolation to transform RGB into CIE LAB can be found in paper [3].

CIE LAB color space is used in this paper as a perceptually uniform space and then the K-L transforms are found in this space. The work is implemented with five groups of color images and in three main steps:

Step1. Transform all the images from the original image color space into CIE LAB color space.

Step2. Compute the covariance matrix in CIE LAB space with all the pixels of all the images in a group.

Step3. Find the three eigenvalues and their corresponding eigenvectors of the covariance matrix and then make the K-L transform with the three eigenvectors.

Among the steps, Step2 and Step3 are actually for principal component analysis (PCA) [4].

Consider $\boldsymbol{X}=\left\{\boldsymbol{x}_{1}, \boldsymbol{x}_{2}, \cdots \boldsymbol{x}_{n}\right\}$ is a collection of pixel samples in the CIE LAB color space. Each sample, $\boldsymbol{x}_{k}=\left(x_{k 1}, x_{k 2}, x_{k 3}\right)^{T}$, has 3 elements, which are 3 components $L^{*}, a^{*}$ and $b^{*}$ respectively. We use these samples to calculate the covariance matrix of $\boldsymbol{X}$, denoted by $S$. 


$$
\boldsymbol{S}=\sum_{k=1}^{n}\left(\boldsymbol{x}_{k}-\boldsymbol{m}\right)\left(\boldsymbol{x}_{k}-\boldsymbol{m}\right)^{T}
$$

where $\boldsymbol{m}$ is the mean vector of the $n$ sample pixels, $\boldsymbol{m}=\frac{1}{n} \sum_{k=1}^{n} \boldsymbol{x}_{k}$.

$\boldsymbol{S}$ is a $3 \times 3$ symmetric matrix, and the solutions of the principal component analysis satisfy the equation: $\boldsymbol{S} \boldsymbol{v}=\lambda \boldsymbol{v}$, where $\lambda$ is one of the 3 eigenvalues of matrix $\boldsymbol{S}$ and $v$ is the corresponding column eigenvector.

For each covariance matrix, we can obtain three eigenvalues $\lambda_{1}, \lambda_{2}, \lambda_{3}$ and their eigenvectors $v_{1}, v_{2}, v_{3}$.

Then, the K-L transform is defined as $\boldsymbol{y}=\boldsymbol{V} \boldsymbol{x}$, where $\boldsymbol{x}$ is a pixel in the CIE LAB space, $\boldsymbol{y}$ is the resultant pixel after transformation, and $\boldsymbol{V}=\left[\boldsymbol{v}_{1}, \boldsymbol{v}_{2}, \boldsymbol{v}_{3}\right]^{T}$.

The samples to make matrix $\boldsymbol{X}$ may be all the pixels of an image or all the pixels of all the images in a group. The computation of the covariance matrix is the most complex work and it has a temporal complexity of $O\left(n^{2}\right)$. A covariance matrix from an image gives a K-L transform that is optimal for the single image. The matrix generated from all the images in a group provides a K-L transform that is optimal for the group images as a whole.

The three eigenvalues are useful if we want to know whether a component is more important than another. The larger an eigenvalue is, the more important its corresponding component is. In this paper, the eigenvector that corresponds to the maximum eigenvalue is called principal eigenvector. By using the principal eigenvector in the $\mathrm{K}-\mathrm{L}$ transform, the most important component can be found. This is helpful for many color image applications, such as color image coding and color image retrieval.

\section{PCA Experiments}

\subsection{Color Images}

In this paper, a database of 5 image groups is used. All of them are downloaded from internet.

1. Architectures: 56 images in JPEG format of Arian architectures in Japan, Iran and Indonesia. Two are shown in Figure 1.

2. Sports: 53 JPEG images of football and soccer games. Two are shown in Figure 2.

3. Animals: 50 JPEG images of feral horses. Two are shown in Figure 3.

4. Plants: 125 JPEG images of many diverse plants. Two are shown in Figure 4.

5. Landscapes: 99 images in bitmap format. Landscape pictures of Greenland. Two are shown in Figure 5.

The images in Group 1, 2 and 5 are downloaded from

Department of Computer Science \& Engineering, University of Washington (http://www.cs.washington.edu /research/imagedatabase/groundtruth/). Those in Group 3 and 4 are downloaded from Electrical Engineering and Computer Sciences, University of California, Berkeley (http://elib.cs.berkeley.edu/photos/).

For the JPEG files, each pixel is converted from YCbCr color space back into RGB color space first during decoding process.
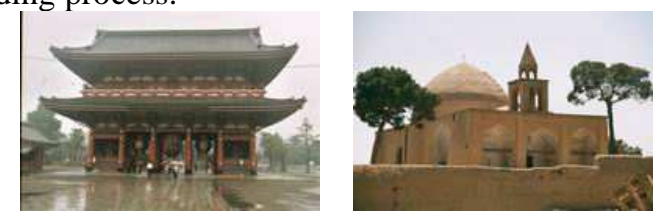

Figure 1. Pictures of the architectures in group 1

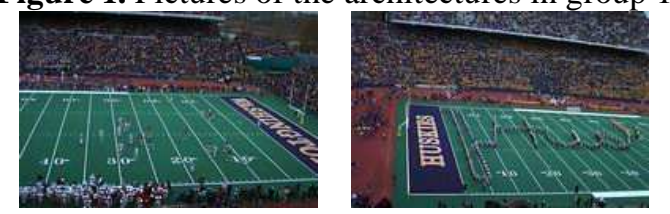

Figure 2. Pictures of a football game in group 2

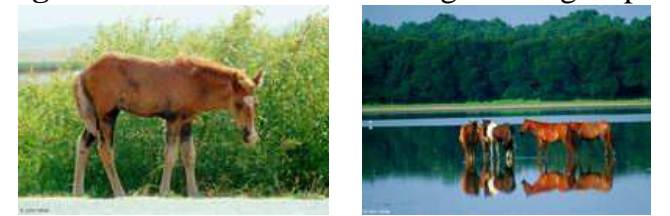

Figure 3. Pictures of feral horses in group 3

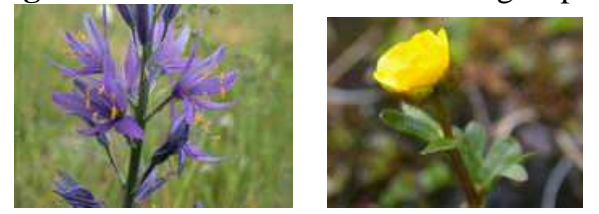

Figure 4.Pictures of plants in group 4

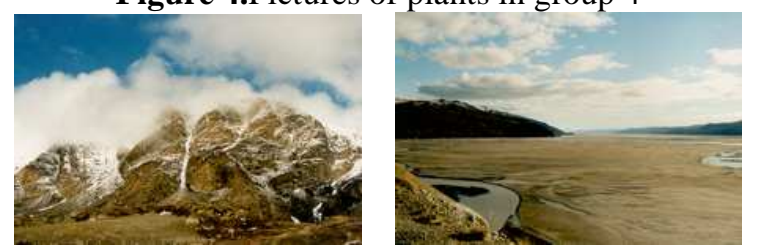

Figure 5. Pictures of landscapes of Greenland in group 5

\subsection{The Optimal Color Transform}

The principal component analysis takes place in the CIE LAB color space, so the eigenvectors and the K-L transform are also in the CIE LAB space. The K-L transform matrix $\boldsymbol{V}$ and three eigenvectors have a relation of $\boldsymbol{V}=\left[\boldsymbol{v}_{1}, \boldsymbol{v}_{2}, \boldsymbol{v}_{3}\right]^{T}$. The eigenvalues are sorted in the magnitude-descending order, and $v_{1}$ is the principal vector.

In order to better understand the proportions between the eigenvalues, we use eigPercent to stand for the percentages of eigenvalues relative to the sum of all three.

We have obtained some very close results with individual image groups and even some individual images. In a group, the principal eigenvector of each single picture is close to the principal eigenvector of its image group.

There is an interesting result that the principal 
eigenvectors of the 5 image groups are all close to $(1,0,0)^{T}$. That means, in the CIE LAB space, the most important component of all the images is in the direction of $L^{*}$.

We also use all the test images to find the general K-L transform for all the groups. The K-L transform matrix (transpose of eigenvectors matrix), eigenvalues and their proportional percentages from all the images in all the groups are:

$$
\begin{gathered}
\boldsymbol{V}=\left[\boldsymbol{v}_{1}, \boldsymbol{v}_{2}, \boldsymbol{v}_{3}\right]^{T}=\left[\begin{array}{rrr}
0.9992 & -0.0301 & 0.0275 \\
-0.0018 & -0.7065 & -0.7077 \\
0.0408 & 0.7070 & -0.7060
\end{array}\right] \\
{\left[\lambda_{1}, \lambda_{2}, \lambda_{3}\right]=10^{11} \times[1.1990,0.5694,0.5060]} \\
\text { eigPercent }=[52.72,25.04,22.25]
\end{gathered}
$$

The K-L transform for all the images in our database also implies that the principal vector in CIE LAB space is very close to the vector $(1,0,0)^{T}$, and the other components are primarily in the AOB plane of the CIE LAB coordinate system, where $\mathrm{O}$ is the origin in CIE LAB space. Both vectors have a rotation angle of about $\pi / 4$ to CIE LAB axes except a sign.

Therefore, through our experiments, an approximate optimal transform can be given as:

$$
\boldsymbol{V}=\left[\begin{array}{rrr}
1 & 0 & 0 \\
0 & \cos (\pi / 4) & \sin (\pi / 4) \\
0 & -\sin (\pi / 4) & \cos (\pi / 4)
\end{array}\right]
$$

It looks so elegant and simple. More importantly, the color space after this transform is still perceptually uniform, and we believe it is a better choice for perceptually uniform color space standard. Since the new space is LAB rotated, we name the transformed space as LAR.

From the proportional percentages between the eignevalues, we can know that the significance between the components in LAR space is roughly $2: 1: 1$. This also looks very nice and very useful in image applications.

The percentages of the three eigenvalues give the information of importance, which can be used in image retrieval or image compression during quantization.

\section{Application in Image Retrieval}

The optimal transform in CIE LAB space has many applications. In this paper, we use 5 groups of images to test color-based image retrieval with different color transforms. Our transform LAR averagely gives better performance than RGB, YCbCr, YUV and $\mathrm{LAB}$ in our experiments.

\subsection{Images for Retrieval}

In order to test the color-based image retrieval performance of LAR comparing to $\mathrm{YCbCr}, \mathrm{YUV}$ and
LAB, we manually classify 120 color images into 6 groups to make our experiments. Each test group has 20 images of visually similar content. Among them, the images of 5 groups are selected from the images we used in the former section. The other 20 images are selected from the images of Cannon Beach, and they are of buildings near the beach. Two of them are showed in Figure 6. They are also downloaded from the website at University of Washington.
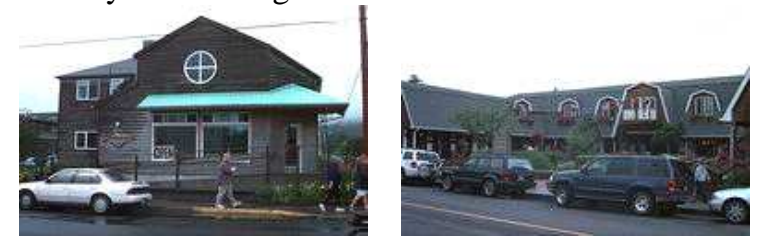

Figure 6. Pictures in Group 6, Cannon Beach buildings

\subsection{Similarity Function}

Intersection of histograms is used as the similarity function for image comparison in this paper.

Suppose the histogram of an image is

$$
H=\left\{(h(0), h(1), \cdots) \mid \sum_{k} h(k)=1\right\}
$$

then the similarity function of two images $I_{1}$ and $I_{2}$ is defined as: $S=\sum_{k} \min \left(h_{1}(k), h_{2}(k)\right)$

It is believed that the larger the value $S$ is, the more similar the two images are. According to this definition, we have $S=1$ if $I_{1}=I_{2}$.

For multi-component images, $H$ is defined by concatenation of the histograms of all the components. For example, if $I$ has three components (RGB or LAR), $I_{C 1}, I_{C 2}, I_{C 3}$, then :

$H=\left\{\left(h_{C 1}(0), h_{C 1}(1), \cdots, h_{C 2}(0), h_{C 2}(1) \cdots h_{C 3}(0), h_{C 3}(1) \cdots\right) \mid \sum_{i} h_{C j}(i)=1, j=1,2,3\right\}$

\subsection{Evaluation}

Let $I_{q}$ be the image in a query, as in [5], we use two numbers to assess our image retrieval answers, precision $p$ and recall $r$, which are widely accepted measures for evaluation of image retrieval. Precision is also called effectiveness and recall of an answer is defined similarly by efficiency in [6].

Precision or effectiveness $p$ is the fraction of the returned images that is indeed relevant for the query. It evaluates the amount of false negative in a recovery, and it increases if the false negative decreases. Recall or efficiency $r$ is the fraction of relevant images that is returned by the query. It evaluates the false positive, and it increases if the false positive decreases. On the ideal occasion, $p$ and $r$ are both equal to the number of the images in a group, 20 in our experiments. By comparison with image $I_{q}$, all the images are sorted in descending order of similarity, $I_{q 1}, I_{q 2}, \cdots I_{q N}$, where $N$ is the total number of images in our image database, 120. That is, if 
$i<j$, then $I_{q i}$ is more similar to $I_{q}$ than to $I_{q j}$. In our experiments, the formulas to find $p$ and $r$ are:

$$
p=\sum_{1 \leq i \leq 20} \operatorname{In}\left(I_{i}, G_{q}\right) \quad r=\min \left\{i \mid \sum_{k \leq i} \operatorname{In}\left(I_{q k}, G_{q}\right)=N_{q}\right\}
$$

where $G$ denotes a image group, $G_{q}$ stands for the image group that includes $I_{q}$, and $N_{q}$ is the number of images in $G_{q}$, and

$$
\operatorname{In}\left(I_{i}, G_{j}\right)= \begin{cases}1 & \text { if } I_{i} \in G_{j} \\ 0 & \text { otherwise }\end{cases}
$$

Ideally, in our experiments, the first 20 similar images are all the images in the same group of the query images, $p=20, r=20$. Unfortunately, this does not occur mostly. Usually, $p$ is smaller than 20 and $r$ is larger than 20 . Therefore, our image retrieval evaluation is based on the average of $p$ and $r$, and they are transformed to percentages in our tables by following formulas.

$$
\begin{gathered}
\text { effectiveness }=100 \times \text { average }(p) / 20 \\
\text { effeciency }=100 \times 20 / \operatorname{average}(r)
\end{gathered}
$$

The color transforms we tested are RGB, YUV, YCbCr, HSV, LAB, and our LAR.

\subsection{Image Retrieval Experiments}

Table 1 and Table 2 list the numbers of effectiveness and efficiency respectively. Note that the values are normalized to percentages.

Table 1 shows that effectiveness of our proposed LAR is averagely better than RGB, YCbCr, YUV and LAB color spaces and a little worse than HSV but comparable.

Table 2 shows that efficiency of LAR is averagely better than all other color spaces, including HSV.

Table 1 Image retrieval effectiveness (\%)

\begin{tabular}{|c|c|l|l|c|c|c|}
\hline Grp & RGB & YCbCr & YUV & HSV & LAB & LAR \\
\hline 1 & 48.75 & 63.75 & 64.00 & 64.25 & $\mathbf{6 6 . 7 5}$ & 62.75 \\
\hline 2 & 83.50 & 88.25 & 89.25 & 95.50 & 92.75 & $\mathbf{9 8 . 5 0}$ \\
\hline 3 & 57.50 & 69.50 & 68.50 & $\mathbf{7 6 . 7 5}$ & 71.50 & 67.75 \\
\hline 4 & 27.75 & 31.00 & 31.00 & 30.25 & 34.00 & $\mathbf{3 4 . 2 5}$ \\
\hline 5 & 49.25 & 41.50 & 42.25 & $\mathbf{6 9 . 7 5}$ & 46.50 & 54.00 \\
\hline 6 & 95.25 & 96.50 & 95.50 & $\mathbf{9 9 . 0 0}$ & 97.00 & $\mathbf{9 9 . 0 0}$ \\
\hline Avg & 60.33 & 65.08 & 65.08 & $\mathbf{7 2 . 5 8}$ & 68.08 & 69.38 \\
\hline
\end{tabular}

Table 2 Image retrieval efficiency $(\%)$

\begin{tabular}{|c|c|l|c|c|c|c|}
\hline Grp & RGB & YCbCr & YUV & HSV & LAB & LAR \\
\hline 1 & 22.75 & 29.18 & 29.24 & 32.03 & $\mathbf{3 2 . 4 1}$ & 29.56 \\
\hline 2 & 53.62 & 56.26 & 55.71 & 76.48 & 71.05 & $\mathbf{9 7 . 5 6}$ \\
\hline 3 & 27.01 & 40.73 & 40.61 & $\mathbf{4 8 . 3 1}$ & 47.00 & 41.15 \\
\hline 4 & 16.88 & 17.29 & 17.30 & 17.91 & 17.69 & $\mathbf{1 8 . 8 9}$ \\
\hline 5 & 24.43 & 19.89 & 20.01 & $\mathbf{3 2 . 8 1}$ & 19.24 & 22.04 \\
\hline 6 & 63.09 & 76.92 & 76.34 & 97.80 & 80.48 & $\mathbf{9 9 . 0 1}$ \\
\hline Avg & 34.63 & 40.05 & 39.87 & 50.89 & 44.65 & $\mathbf{5 1 . 3 7}$ \\
\hline
\end{tabular}

Since the LAR is obtained by a rotation of $\pi / 4$ about the $\mathrm{L}^{*}$ axis, the difference between LAR and LAB is not large. However, we can still find that LAR is superior to LAB in the most cases. As the table 2 shows, the average efficiency of HSV is larger than that of LAB, but smaller than LAR. Therefore, the simple rotation transform is crucial.

\section{Conclusions}

Principal component analysis is applied to obtain K-L transforms for the images of 5 image groups in the perceptually uniform color space, CIE LAB. The eigenvectors of the 5 image groups are close. The principal eigenvectors corresponding to the maximum eigenvalues are all approximately close to the direction of $L^{*}$. The other two eigenvectors are almost perpendicular to the $L^{*}$ direction.

With a number of test images of diverse content, a general optimal K-L transform is obtained and can be used as a fixed optimal color transform for all the images. Such a transform LAR as we presented in 3.2 is actually very nice and simple.

With a fixed and optimal color transform and a faster color space transform between RGB and CIE LAB, a wonderful transform can be found for real-time applications.

The color space K-L transform for an image group is useful for image retrieval and image coding as preprocessing. When an image is given in practice, an appropriate fixed optimal color transform can be applied after we simply recognize what class the image should belong to.

The approximately optimal K-L transform presented in Section 3, LAR, can be used as a fixed color transform in color-based image retrieval. Better results are obtained in LAR space than in RGB, YCbCr, YUV, HSV and LAB spaces. Compared with LAB space, LAR is more effective and efficient for high accurate retrieval.

\section{References}

[1] J. M. Kasson and W. Plouffe, "An Analysis of Selected Computer Interchange Color Spaces", ACM Transactions on Graphics, Vol. 11, No. 4, 1992, pp. 373405.

[2] Charles Poynton, "Frequently Asked Questions about Color", www.inforamp.net/ poynton, 1999.

[3] K. Asakawa and H. Sugiura, "High-precision color transformation system", IEEE Transactions on Consumer Electronics, Vo.41, No.2, May 1995, pp.304-312

[4] R.O. Duda, P.E. Hart and D.G. Stork, "Pattern Classification", Wiley InterScience, 1999.

[5] A.W.M. Smeulders, M. Worring, S. Santini, A. Gupta, and R,. Jain, "Content-Based Image Retrieval at the End of Early Years," IEEE Trans. on Pattern Analysis and Machine Intelligence, vol. 22, no. 12, Dec. 2000, pp. 1349-1380.

[6] E. Mathias and A. Conci, "Comparing the influence of color spaces and metrics in content-based image retrieval", Int. Sym. on Computer Graphics, Image Processing, and Vision, 1998. SIBGRAPI'98, pp. 371-378 